\title{
Sustainability of a Physical Activity and Nutrition Program for Seniors
}

Maria Pasalich, BSc, School of Public Health, Curtin University

Andy H. Lee, PhD, School of Public Health, Curtin University

Jonine Jancey, PhD, School of Public Health, Curtin University

Linda Burke, MPH, School of Public Health, Curtin University

Peter Howat, PhD, Centre for Behaviour Research in Cancer Control, Curtin University

\section{Corresponding author:}

Maria Pasalich

Address: School of Public Health, Curtin University, G.P.O. Box U 1987, Perth, Western

Australia, 6845.

Telephone: +61-8-92664535

Fax: +61-8-92662958

Email: maria.pasalich@curtin.edu.au 
Keywords: program evaluation, exercise, diet, older adult, health promotion

ABSTRACT

Introduction: There is insufficient evidence regarding the sustainability of physical activity and nutrition interventions for older adults. This prospective cohort study aimed to determine the long-term impact of a low cost, home-based physical activity and nutrition program for insufficiently active 60 to 70 year olds, who resided in suburbs with low to medium socioeconomic status.

Methods: A follow-up survey was conducted six months after program completion via computer-assisted telephone interviewing. The International Physical Activity Questionnaire and the Fat and Fibre Barometer were used to measure physical activity levels and dietary behaviours, respectively. Self-reported height, weight, waist and hip circumferences were obtained. Changes over three time points of data collection (baseline, post-program, follow up) and differences between the intervention and control groups were assessed. The use of program materials was also evaluated.

Results: Sustained improvements were observed for the intervention group in strength exercises, fibre intake, fat intake, fat avoidance, body mass index and waist-to-hip ratio. Mean walking time decreased below baseline levels for both groups. At post-program, the intervention participants had increased time spent in moderate activity $(\mathrm{p}>.05)$, which declined at follow-up $(\mathrm{p}<.05)$. Conclusion: This low-cost physical activity and nutrition intervention resulted in sustained improvements in dietary outcomes and overall short-term gains in physical activity. Future studies for older adults are recommended to investigate behavioural barriers as well as booster interventions targeting areas of physical activity.

(word count: 230) 


\section{Introduction}

Older adults, a growing segment of the population, are not participating in sufficient amounts of physical activity, nor are they meeting the recommended daily intake of fruit and vegetables (1). Regular physical activity and a nutritionally balanced diet can lead to a range of health benefits and play a preventative role for many chronic health conditions such as type 2 diabetes, cardiovascular disease and various cancers (1-3).

Interventions aimed at increasing physical activity levels and encouraging the adoption of a healthy diet for seniors have generated mixed results. A 2-year randomised controlled trial found no significant differences in physical activity between the exercise and comparison groups 12 months after intervention and at 24-month follow-up (4), while the diet intervention participants increased their consumption of fruit and vegetables over the study period (5). Morey and colleagues observed significant improvements in self-reported dietary behaviours, physical activity and weight loss for the intervention group relative to the controls at 12 months postprogram (6). Another study concluded that an exercise training or weight-management (diet) program can improve the health of obese older adults, whereas a combination of both interventions may have a synergistic effect (7).

Although these interventions have provided insight into their impact, follow-up evaluation after a non-contact period is an area of program sustainability that has been largely ignored. In addition, most intervention studies were conducted using motivated volunteers (7-9) and focused on older 
age groups ( $\geq 70$ years) (6-8). Seniors who are sedentary or underactive and those living in lower socioeconomic status (SES) suburbs are rarely targeted.

The present study aimed to determine the long-term impact of a low cost, home-based physical activity and nutrition intervention for seniors. Changes in self-reported physical activity levels, dietary behaviours, and anthropometric measures over time were investigated, as well as the use of program materials.

\section{Methods}

\section{Study design and data collection procedure}

Physical Activity and Nutrition for Seniors (PANS) was a 6-month, low-cost, home-based, semitailored physical activity and nutrition intervention that incorporated goal setting and social support (10). The target group participants were insufficiently active older adults (aged 60 to 70 years) randomly drawn from low and medium SES suburbs in the Perth metropolitan area of Western Australia. Participants received a range of supportive resources including written materials, pedometer, and resistance band, together with email and telephone contact (10).

In this prospective cohort study, follow-up data were collected in 2011 from participants 6 months after the PANS intervention concluded. The questionnaire was completed via computerassisted telephone interviewing (CATI) to reduce attrition that occurred during the first two 
phases of data collection, in which baseline and post-program questionnaires were distributed by postal mail. Two weeks prior to the CATI survey, a reminder postcard was sent to each participant. It explained the purpose of the forthcoming telephone call and encouraged participants to record their measurements (i.e. height, weight, waist circumference and hip circumference) on the postcard in preparation for their telephone interview. Participants were subsequently contacted by a trained interviewer who entered the responses directly into a database. Minimal burden was placed on participants, with each interview being completed within 15 to 20 minutes. There were other advantages of the CATI approach. Participants could be recontacted at a time that suited them, questions could be clarified without delay, and the process ensured no missing data. Participants were phoned a maximum of five times before being classified as non-respondents. All participants were posted a lottery ticket as an incentive to complete the telephone survey.

To gather information on the role of the program resources, a subsample of the intervention group $(n=20)$ were telephoned by the first author on a separate occasion for qualitative feedback. The study was approved by the Curtin University Human Research Ethics Committee (approval number HR 186/2008) and verbal consent was obtained from all participants during initial recruitment. A copy of the questionnaire used in the CATI survey is available from the first author upon request.

\section{Participants}


Details of participant recruitment at baseline were extensively described elsewhere (10). The cohort under consideration comprised of participants who had completed both baseline and postprogram questionnaires and involved in either the intervention $(n=176)$ or the control $(n=198)$ group.

\section{Measuring instruments}

The follow-up questionnaire was modified slightly from previous questionnaires used at baseline and post-program. It was shortened to focus on physical activity levels, dietary behaviours, anthropometric measures and demographic characteristics.

Physical activity was assessed using the short form of the International Physical Activity Questionnaire (IPAQ) (10). The instrument measured frequency (days per week) and duration (minutes per day) of walking and moderate-intensity activity. A strength exercise question taken from the American Heart Association (11) was added, but vigorous activity was not assessed due to the low number of elderly subjects participating in this type of activity.

Dietary behaviours were measured using a modified version of the Fat and Fibre Barometer (FFB), a brief dietary assessment tool specifically developed to evaluate self-reported fat and fibre related food behaviours (12). Four questions on fibre-related intake (i.e. fruit, vegetables, legumes, cereal) were summed to create a 'fibre intake' score (range 0-28), whereas three 
questions on fat-related intake (i.e. fried food, pastries, take-away) were summed to create a 'fat intake' score (range 0-21), with higher scores indicating greater intake. Six questions relating to the avoidance of fat (e.g. "how often do you trim all visible fat off the meat?") were summed to create a 'fat avoidance' score (range 6-30), with a higher score indicating increased fat avoidance.

Anthropometric measurements recorded were self-reported height, weight, and waist and hip circumferences. Body mass index (BMI) and waist-to-hip ratio (WHR) were then calculated (13).

To assess the usage of program materials at follow-up, all intervention group participants were asked an additional question: "Are you currently using any of the following PANS materials: booklet, exercise chart, pedometer, and resistance band?" To provide further insight, 20 PANS participants were purposely selected and interviewed based on their responses relating to supportive resources at post-program and follow-up. Participants who answered 'agree' or 'strongly agree' to the statement "I will use the PANS materials in six months time" (at postprogram) but then answered "no" to the items covering use of program materials at least three times (at follow-up) were contacted. The information would enable the identification of reasons or barriers for non-use.

\section{Statistical analysis}


Demographic and health characteristics were summarised using descriptive statistics. Paired sample t-tests were performed to determine if significant changes in the primary outcomes had occurred between the three time points, $\mathrm{t} 1$ (baseline), $\mathrm{t} 2$ (post-program) and $\mathrm{t} 3$ (follow-up), of data collection. Independent samples t-tests were conducted to determine whether significant differences in primary outcomes arose between intervention and control groups. Frequencies were obtained in relation to usage of program materials. All statistical analyses were undertaken using the SPSS package version 18, at the 0.05 level of significance, for the cohort with complete data at all three time points.

\section{Results}

\section{Demographics}

The follow-up survey was completed by 162 intervention participants and 187 controls, giving an overall response rate of 93\%; see Figure 1. There were no significant differences in demographic characteristics between participants in either group and those lost to follow-up. Table 1 shows the demographic profile of intervention and control participants at follow-up. No significant

differences in demographic characteristics were found between the two groups $(p>.05)$. The mean age of participants was 65 (standard deviation, 3.06) years. The ratio of males to females was fairly equal. Approximately $42 \%$ of participants were still employed, and the majority (77\%) lived with a partner.

\section{Physical activity level}


Table 2 presents the three physical activity outcomes over the three time points. No significant differences were found between the intervention and control groups at all three time points. Baseline versus post-program paired t-tests indicated significant improvements for the intervention group in terms of strength activity, whilst the control group showed little change ( $p$ > .1). Paired t-tests for post-program versus follow-up indicated a significant decrease in moderate activity across both groups. Over this 6-month follow-up period, both groups reduced their mean walking time ( $\mathrm{p}=.02$ for control). Mean strength activity also decreased $(\mathrm{p}>.1)$, yet remained above baseline level for the intervention group.

\section{Dietary behaviour}

Results of the three dietary outcomes are presented in Table 2. Significant increases in fat avoidance and fibre intake mean scores were observed for the intervention group at post-program $(\mathrm{p}<.001)$. Their mean scores were also significantly higher than the corresponding mean scores for the controls $(p<.05)$. A significant reduction in the fat intake mean score was found at both post-program $(\mathrm{p}<.05)$ and follow-up $(\mathrm{p}<.001)$ in the intervention group, and at follow-up only in the control group $(\mathrm{p}<.001)$.

\section{Anthropometry}

The anthropometric outcomes, BMI and WHR, are given in Table 3. At follow-up, the intervention participants had a significantly lower mean BMI relative to both post-program and 
baseline $(\mathrm{p}<.01)$ and maintained the initial reduction in mean WHR. No significant changes in anthropometric outcomes were recorded for controls.

\section{Use of program materials}

Almost half the intervention group (45\%) were still using the pedometer at follow-up, just over one third (35\%) were using the exercise chart, whilst the booklet and resistance band were utilised to a lesser extent (29\% and 27\%, respectively). Overall, $35.8 \%$ of the intervention participants were not using any of the program materials at follow-up.

Feedback from telephone interviews of selected participants were summarised. The most common reason cited for discontinued use of the program materials (by seniors who expressed their intention of usage at post-program) was "busy/lack of time”, followed by "injury/soreness/ illness".

\section{Discussion}

The objective of this prospective cohort study was to assess the sustainability of the PANS intervention at 6 months follow-up. The results indicate short-term gains in physical activity levels post-program (with the exception of strength) but sustained improvements in dietary 
behaviours in the longer term, after the seniors were subjected to a low-cost, home-based physical activity and nutrition intervention.

\section{Physical activity}

The PANS intervention was effective in increasing time spent doing strength activities, and such improvement was sustained over the long-term (6 months follow-up). This may be attributed to the provision of resistance bands and exercise charts to aid with the home-based exercise program. A 12-month home-based study that provided participants with similar resources also reported increases in strength training duration post-program (6).

Moderate activity declined after the 6-month non-contact period, to levels below baseline ( $\mathrm{p}<$ .05). Similar decline in walking time was observed though statistically non-significant ( $\mathrm{p}>.05)$. These outcomes may be related to the overall low use of the program materials, as over one third of PANS participants reported not using any of the program resources at follow-up. There were no significant differences in self-reported physical activity levels between intervention and control groups at post-program and follow-up. Similar findings were reported in a previous study with intervention and follow-up periods lasting 12 months (4), where physical activity remained stable over the 24-month period for both control and intervention groups.

\section{Dietary behaviour}


The PANS intervention successfully increased fibre intake, decreased fat intake, and improved fat avoidance behaviours at post-program, and most importantly, maintained these behaviours at follow-up. Therefore, it appears that the PANS intervention approach, targeting specific dietary behaviours, can lead to positive long-term dietary changes in older adults. The finding is consistent with a previous study which reported that intervention participants sustained a significantly lower saturated fat intake compared to controls at 12-months post-program (6).

\section{Anthropometric measures}

The PANS intervention was effective in reducing WHR at post-program, and such reduction was maintained at follow-up. WHR is an indicator of abdominal fat (14). Studies have shown that this region is responsive to weight loss interventions i.e. a preferential loss of abdominal fat is associated with a modest weight loss $(15,16)$. BMI fell slightly among the intervention group at post-program, with a further significant reduction observed at follow-up. These sustained decreases are probably due to continued participation in strength exercises and improvements in fat and fibre-related dietary behaviours. Previous studies evaluating weight loss after a 12-month period have noted similar positive results $(6,17)$.

\section{Implications}

The follow-up results showed an overall decrease in physical activity levels over time, which is consistent with the literature once the interventions and incentives have been removed (18). The finding supports the need for further contact following the conclusion of the original intervention 
by way of a booster program. Minimal intervention booster programs have been found successful in enhancing the sustainability of the original intervention $(19,20)$. A 3-month home-based physical activity booster program consisting of a pedometer, print materials, and motivational phone calls, conducted 12 months after a 6-month walking intervention successfully raised the weekly mean time spent walking for recreation (19). Similar follow-up booster programs in the area of exercise therapy have positively influenced maintenance of post-treatment outcomes (20).

Older adults tend to be vulnerable to certain barriers when attempting to make changes to physical activity behaviours (21). Barriers such as poor health (22), neighbourhood walkability (23), weather (24), as well as individual beliefs and attitudes (25), must be taken into consideration when designing physical activity programs. Furthermore, social support to enhance motivation may be relevant during the maintenance period following a physical activity program (26). If the use of program materials was maintained at 6 months follow-up, physical activity outcomes might have been maintained closer to post-program levels. Reasons for discontinued use of the program materials included "forgot", "exercise not a priority" and "didn't think I had to continue using them". These issues can be addressed through further contact by way of reminder phone calls and newsletters. A booster program would provide the impetus to overcome some of these obstacles and potentially reactivate their positive attitude towards physical activity. 
Long-term behaviour change is the ultimate goal of any health promotion intervention. With an ageing population prone to chronic diseases and becoming increasingly obese, it is important to conduct follow-up evaluation of programs that aim to improve the health status of seniors.

\section{Limitations}

In addition to selection bias of the voluntary participants, all outcomes were self-reported, therefore reporting bias may affect their validity due to overestimation. Nevertheless, the main concern was the change in health behaviours over time. It is reliability (rather than validity) which is of greater concern, so that the self-report data should be sufficiently reliable for examining behavioural changes $(12,27-29)$. Usage of program materials could have been examined in more detail, but our priority was to focus on the primary outcomes in order to minimise participant burden. It is also possible that the sustained improvements among the PANS participants were influenced by the Hawthorne effect. However, the attention given to participants throughout the intervention was both planned and deliberate.

Although several physical activity and nutrition randomised control trials have been developed for older adults, levels of behavioural maintenance in the longer term after program completion has seldom been investigated. Whilst sustained improvements in fat and fibre-related dietary behaviours were found after the home-based PANS intervention, increases in physical activity levels at the end of the 6-month intervention were mostly short-term. Future studies are recommended to investigate the barriers affecting seniors' participation in physical activity 
during this maintenance period of behaviour change. In order to prevent chronic diseases, older adults need long-term support and motivation to make beneficial lifestyle changes. Maintaining contact with participants via a booster program is necessary to enable these newly acquired behaviours to become habitual.

(word count: 2572) 


\section{Acknowledgements}

This research was partially supported by a National Health and Medical Research Council

(NHMRC) grant. The authors are grateful to the seniors who participated in this study. 


\section{References}

1. National Center for Chronic Disease Prevention and Health Promotion. Nutrition and physical activity - helping people choose healthy eating and active living. Atlanta (GA): Division of Nutrition, Physical Activity, Obesity; 2011.

http://www.cdc.gov/chronicdisease/resources/publications/aag/pdf/2011/Nutrition-and-PhysActivity-AAG_WEB_PDF.pdf. Accessed December 22, 2011.

2. Bellow A, Epstein J, Parikh-Patel A. Lifestyle behaviors associated with secondary prevention of coronary heart disease among California adults. Prev Chronic Dis 2011;8(2):A31. http://www.cdc.gov/pcd/issues/2011/mar/10_0047.htm. Accessed [December 22, 2011].

3. Miller R, Sales A, Kopjar B, Fihn S, Bryson C. Adherence to heart-healthy behaviors in a sample of the U.S. population. Prev Chronic Dis 2005;2(2):A18.

http://www.cdc.gov/pcd/issues/2005/apr/04_0115.htm. Accessed [December 22, 2011].

4. Greaney M, Riebe D, Ewing Garber C, Rossi J, Lees F, Burbank P, et al. Long-term effects of a stage-based intervention for changing exercise intentions and behavior in older adults. Gerontologist 2008;48(3):358-67.

5. Greene G, Fey-Yensan N, Padula C, Rossi S, Rossi J, Clark P. Change in fruit and vegetable intake over 24 months in older adults: results of the SENIOR project intervention. Gerontologist 2008;48(3):378-87.

6. Morey M, Snyder D, Sloane R, Cohen H, Peterson B, Hartman T, et al. Effects of homebased diet and exercise on functional outcomes among older, overweight long-term cancer survivors. JAMA 2009;301(18):1883-91. 
7. Villareal D, Chode S, Parimi N, Sinacore D, Hilton T, Armamento-Villareal R, et al. Weight loss, exercise, or both and physical function in obese older adults. N Engl J Med 2011;364(13):1218-29.

8. Clark P, Rossi J, Greaney M, Riebe D, Greene G, Saunders S, et al. Intervening on exercise and nutrition in older adults: the Rhode Island SENIOR project. J Aging Health 2005;17(6):753-78.

9. Mills K, Stewart A, King A, Roihz K, Sepsis P, Ritter P, et al. Factors associated with enrollment of older adults into a physical activity promotion program. J Aging Health 1996;8(1):96-113.

10. Burke L, Jancey J, Howat P, Lee A, Kerr D, Shilton T, et al. Physical activity and nutrition program for seniors (PANS): protocol of a randomized controlled trial. BMC Public Health 2010;10(751).

11. Haskell W, Lee I. Physical activity and public health. Updated recommendation for adults from the American College of Sports Medicine and the American Heart Association. Circulation 2007;116:1081-93.

12. Wright J, Scott J. The Fat and Fibre Barometer, a short food behaviour questionnaire: reliability, relative validity and utility. Nutr Diet 2000;57(1):33-9.

13. Visscher T, Snijder M, Seidell J. Epidemiology: definition and classification of obesity. In: Kopelman PG, Caterson ID, Dietz WH, editors. Clinical Obesity in Adults and Children. Oxford: John Wiley \& Sons; 2009. p. 5-6.

14. Rogers M. Preexercise and Health Screening. In: Jones CJ, Rose DJ, editors. Physical activity instruction of older adults. Champaign (IL): Human Kinetics; 2005. p. 62. 
15. Mayo M, Grantham J, Balasekaran G. Exercise-induced weight loss preferentially reduces abdominal fat. Med Sci Sports Exerc 2003;35(2):207-13.

16. Dengel D, Hagberg J, Coon P, Drinkwater D, Goldberg A. Comparable effects of diet and exercise on body composition and lipoproteins in older men. Med Sci Sports Exerc 1994;26(11):1307-15.

17. Witham M, Avenell A. Interventions to achieve long-term weight loss in obese older people. Age Ageing 2010;39(2):176-84.

18. Marcus B, Williams D, Dubbert P, Sallis J, King A, Yancey A, et al. Physical activity intervention studies. Circulation 2006;114(24):2739-52.

19. Jancey J, Lee A, Howat P, Burke L, Leong C, Shilton T. The effectiveness of a walking booster program for seniors. Am J Health Promot 2011;25(6):363-7.

20. Pisters M, Veenhof C, van Meeteren N, Ostelo R, de Bakker D, Schellevis F, et al. Long Term effectiveness of exercise therapy in patients with osteoarthritis of the hip or knee: a systematic review. Arthritis Care Res 2007;57(7):1245-53.

21. Brawley L, Rejeski W, King A. Promoting physical activity for older adults: the challenges for changing behavior. Am J Prev Med 2003;25(3, Supplement 2):172-83.

22. Jancey J, Clarke A, Howat P, Maycock B, Lee A. Perceptions of physical activity by older adults: a qualitative study. Health Educ J 2009;68(3):196-206.

23. Owen N, Cerin E, Leslie E, duToit L, Coffee N, Frank L, et al. Neighborhood walkability and the walking behavior of Australian adults. Am J Prev Med 2007;33(5):387-95.

24. Belza B, Walwick J, Shiu-Thornton S, Schwartz S, Taylor M, LoGerfo J. Older adult perspectives on physical activity and exercise: voices from multiple cultures. Prev Chronic Dis 
2004;1(4). http://www.cdc.gov/pcd/issues/2004/oct/04_0028.htm. Accessed [September 14, 2011].

25. Lee L, Arthur A, Avis M. Using self-efficacy theory to develop interventions that help older people overcome psychological barriers to physical activity: a discussion paper. Int J Nurs Stud 2008;45(11):1690-9.

26. Oka R, King A, Young D. Sources of social support as predictors of exercise adherence in women and men ages 50 to 65 years. Womens Health 1995;1(2):161-75.

27. Craig C, Marshall A, Sjöström M, Bauman A, Booth M, Ainsworth B, et al. International physical activity questionnaire: 12-country reliability and validity. Med Sci Sports Exerc 2003;35(8):1381-95.

28. Stommel M, Schoenborn C. Accuracy and usefulness of BMI measures based on selfreported weight and height: findings from the NHANES \& NHIS 2001-2006. BMC Public Health $2009 ; 9(421)$

29. Spencer E, Roddam A, Key T. Accuracy of self-reported waist and hip measurements in 4492 EPIC-Oxford participants. Public Health Nutr 2004;7(6):723-7. 
Table 1. Demographic profile of intervention participants and controls

\begin{tabular}{|l|c|c|c|}
\hline Variable & $\begin{array}{c}\text { Intervention group } \\
(\mathbf{n = 1 6 2})\end{array}$ & $\begin{array}{c}\text { Control group } \\
(\mathbf{n = 1 8 7})\end{array}$ & $\mathbf{p}$ \\
\hline Age: mean (SD) years & $65.81(2.94)$ & $65.65(3.17)$ & .62 \\
\hline Gender: male & $84(51.9 \%)$ & $92(49.2 \%)$ & .62 \\
\hline $\begin{array}{l}\text { Relationship status: } \\
\text { with partner }\end{array}$ & $119(73.5 \%)$ & $149(79.7 \%)$ & .17 \\
\hline $\begin{array}{l}\text { Education: } \\
\text { high school and above }\end{array}$ & $154(95.1 \%)$ & $172(92.0 \%)$ & .57 \\
\hline $\begin{array}{l}\text { Work status: } \\
\text { still employed }\end{array}$ & $68(42.0 \%)$ & $77(41.2 \%)$ & .88 \\
\hline $\begin{array}{l}\text { Presence of } \\
\text { co-morbidity }\end{array}$ & $119(73.5 \%)$ & $131(70.1 \%)$ & .48 \\
\hline
\end{tabular}

SD: standard deviation

${ }^{a}$ Co-morbidity defined as the presence of at least one of nine health conditions 
Table 2. Physical activity and dietary outcomes of intervention participants and controls at baseline (t1), post-program (t2) and follow-up (t3)

\begin{tabular}{|c|c|c|c|c|}
\hline Outcome & Time point & Intervention group & Control group & $\mathbf{p}^{\mathbf{a}}$ \\
\hline \multirow{5}{*}{$\begin{array}{l}\text { Walking time } \\
\text { (minutes per week): } \\
\text { mean (SD) }\end{array}$} & $\mathrm{t} 1$ & $314.9(644.9)$ & $299.0(513.3)$ & .80 \\
\hline & $\mathrm{t} 2$ & $319.3(596.8)$ & $285.9(503.1)$ & .57 \\
\hline & $\mathrm{t} 3$ & $247.3(324.8)$ & $215.1(306.2)$ & .34 \\
\hline & $p^{b}$ & .93 & .66 & \\
\hline & $p^{c}$ & .10 & .02 & \\
\hline \multirow{5}{*}{$\begin{array}{l}\text { Moderate physical } \\
\text { activity (minutes per } \\
\text { week): mean (SD) }\end{array}$} & $\mathrm{t} 1$ & $155.4(284.7)$ & $197.2(393.9)$ & .26 \\
\hline & $\mathrm{t} 2$ & $205.8(411.4)$ & $193.7(264.2)$ & .74 \\
\hline & $\mathrm{t} 3$ & $110.9(162.0)$ & $128.5(188.2)$ & .36 \\
\hline & $\mathrm{p}^{\mathrm{b}}$ & .11 & .89 & \\
\hline & $\mathrm{p}^{\mathrm{c}}$ & .004 & .003 & \\
\hline \multirow{5}{*}{$\begin{array}{l}\text { Strength activity } \\
\text { (minutes per week): } \\
\text { mean (SD) }\end{array}$} & $\mathrm{t} 1$ & $25.3(88.9)$ & $39.4(169.3)$ & .34 \\
\hline & $\mathrm{t} 2$ & $47.2(109.2)$ & $37.8(119.7)$ & .45 \\
\hline & $\mathrm{t} 3$ & $36.9(128.3)$ & $23.4(63.2)$ & .23 \\
\hline & $\mathrm{p}^{\mathrm{b}}$ & .02 & .91 & \\
\hline & $\mathrm{p}^{\mathrm{c}}$ & .44 & .12 & \\
\hline \multirow{5}{*}{$\begin{array}{l}\text { Fat intake score: } \\
\text { mean }(\mathrm{SD})^{\mathrm{d}}\end{array}$} & $\mathrm{t} 1$ & $1.90(2.03)$ & $1.50(1.59)$ & .04 \\
\hline & $\mathrm{t} 2$ & $1.67(1.62)$ & $1.62(1.90)$ & .75 \\
\hline & $\mathrm{t} 3$ & $1.24(1.70)$ & $1.14(1.48)$ & .55 \\
\hline & $\mathrm{p}^{\mathrm{b}}$ & .05 & .28 & \\
\hline & $p^{c}$ & .001 & $<.001$ & \\
\hline \multirow{5}{*}{$\begin{array}{l}\text { Fat avoidance score: } \\
\text { mean }(\mathrm{SD})^{\mathrm{e}}\end{array}$} & $\mathrm{t} 1$ & $21.09(4.69)$ & $21.11(4.74)$ & .96 \\
\hline & $\mathrm{t} 2$ & $22.35(4.44)$ & $21.10(4.85)$ & .01 \\
\hline & $\mathrm{t} 3$ & $22.14(5.08)$ & $21.28(4.81)$ & .11 \\
\hline & $\mathrm{p}^{\mathrm{b}}$ & $<.001$ & .96 & \\
\hline & $p^{c}$ & .51 & .54 & \\
\hline \multirow{5}{*}{$\begin{array}{l}\text { Fibre intake score: } \\
\text { mean }(\mathrm{SD})^{\mathrm{f}}\end{array}$} & $\mathrm{t} 1$ & $16.21(5.04)$ & $15.43(5.51)$ & .17 \\
\hline & $\mathrm{t} 2$ & $17.18(4.60)$ & $15.97(5.29)$ & .02 \\
\hline & $\mathrm{t} 3$ & $17.45(4.17)$ & $16.57(5.04)$ & .07 \\
\hline & $\mathrm{p}^{\mathrm{b}}$ & .001 & .05 & \\
\hline & $p^{c}$ & .39 & .08 & \\
\hline
\end{tabular}

SD: standard deviation

a intervention versus control using independent samples t-test

${ }^{b} \mathrm{t} 1$ versus $\mathrm{t} 2$ using paired samples t-test

${ }^{c} \mathrm{t} 2$ versus $\mathrm{t} 3$ using paired samples t-test

d range: 0-21 (lower scores represent more favourable responses)

${ }^{\mathrm{e}}$ range: 6-30 (higher scores represent more favourable responses)

${ }^{\mathrm{f}}$ range: 0-28 (higher scores represent more favourable responses) 
Table 3. Anthropometric outcomes of intervention participants and controls at baseline (t1), post-program (t2) and follow-up (t3)

\begin{tabular}{|l|c|c|c|c|}
\hline Outcome & Time point & Intervention group & Control group & $\mathbf{p}^{\mathbf{a}}$ \\
\hline $\begin{array}{l}\text { Body mass index } \\
\left(\mathrm{kg} / \mathrm{m}^{2}\right): \text { mean }(\mathrm{SD})\end{array}$ & $\mathrm{t} 1$ & $27.63(4.42)$ & $27.32(4.62)$ & .53 \\
\hline & $\mathrm{t} 2$ & $27.54(4.42)$ & $27.19(4.44)$ & .46 \\
\hline & $\mathrm{t} 3$ & $27.27(4.23)$ & $27.20(4.50)$ & .88 \\
\hline & $\mathrm{p}^{\mathrm{b}}$ & .24 & .20 & .93 \\
\hline $\begin{array}{l}\text { Waist-to-hip ratio: } \\
\text { mean (SD) }\end{array}$ & $\mathrm{p}^{\mathrm{c}}$ & .003 & $0.925(0.093)$ & .50 \\
\hline & $\mathrm{t}$ & $0.932(0.088)$ & $0.923(0.088)$ & .58 \\
\hline & $\mathrm{t} 2$ & $0.918(0.085)$ & .67 & .93 \\
\hline & $\mathrm{t} 3$ & $0.921(0.088)$ & $.922(0.091)$ & \\
\hline & $\mathrm{p}^{\mathrm{b}}$ & .003 & .52 & \\
\hline
\end{tabular}

SD: standard deviation

a intervention versus control using independent samples t-test

${ }^{b} t 1$ versus $t 2$ using paired samples t-test

${ }^{c} \mathrm{t} 2$ versus $\mathrm{t} 3$ using paired samples t-test 


\section{Figure legend}

Figure 1. Follow-up of intervention participants and controls who had completed both baseline and post-program questionnaires. 